\title{
Metalloenzyme structures in a shot
}

Researchers study the structure of the metalloenzyme photosystem II by applying femtosecond $\mathrm{X}$-ray pulses to simultaneously record $X$-ray diffraction and $\mathrm{X}$-ray emission spectroscopy data.

The X-ray free-electron laser at Linac Coherent Light Source at SLAC National Accelerator Laboratory produces intense, femtosecond X-ray pulses. This offers the unique ability to collect $\mathrm{X}$-ray diffraction data at room temperature before radiation damage occurs, a technique called 'diffraction before destruction' The method is generating much excitement in the structural biology community for its ability to produce high-quality data from protein microcrystals, which are easier to grow than the large crystals needed for traditional X-ray diffraction.

Researchers at Lawrence Berkeley National Laboratory and SLAC recently reported a new use of the X-ray free-electron laser to simultaneously record X-ray diffraction data and X-ray emission spectroscopy data. This method is well-suited to study both protein structures and the electronic structures of catalytic metal centers found in metalloenzymes. In work led by Junko Yano and Vittal Yachandra of Lawrence Berkeley National Laboratory, and Uwe Bergmann of SLAC, researchers applied the method to study the metalloenzyme photosystem II (PS II), a light-driven membrane protein complex found in green plants containing a catalytic $\mathrm{Mn}_{4} \mathrm{CaO}_{5}$ cluster involved in dioxygen production.

"In order to combine crystallography with spectroscopy, we designed an energydispersive emission spectrometer that could collect the entire spectrum on a shot by shot basis from the X-ray laser," say Yano, Yachandra and Bergmann in a joint statement. However, they note that there were many technical challenges to implement the design and apply it to study PS II, which required the combined efforts of a large team of scientists with a wide range of expertise.

The $\mathrm{Mn}_{4} \mathrm{CaO}_{5}$ cluster of PS II is highly sensitive to radiation damage; the scientists point out that it was not obvious that they would be able to obtain electronic structure information even using femtosecond laser pulses. They were thus gratified when the integrated X-ray diffraction-emission spectroscopy approach yielded electronic structure data for both the dark state and the first illuminated state of the $\mathrm{Mn}_{4} \mathrm{CaO}_{5}$ cluster. In the future, they plan to study the intermediate states of this cluster in real time and hope to visualize the formation and release of the dioxygen molecule.

Allison Doerr

RESEARCH PAPERS

Kern, J. et al. Simultaneous femtosecond X-ray spectroscopy and diffraction of photosystem II at room temperature. Science advance online publication (14 February 2013). 